\title{
IMPACTO DOS RESULTADOS DAS PESQUISAS EM ENFERMAGEM NA PRÁTICA PROFISSIONAL
}

\author{
Valéria Lerch Lunardi ${ }^{1}$,Wilson Danilo Lunardi Filho ${ }^{2}$, Rosemary Silva da Silveira ${ }^{3}$, Alacoque Lorenzini Erdmann",
} Rosalina Partezani Rodrigues ${ }^{5}$,Graziele de Lima Dalmolin ${ }^{6}$

\begin{abstract}
RESUMO: Frente à preocupação com os resultados da pesquisa na enfermagem, temos como objetivo refletir sobre seu impacto na prática profissional. Para tal, abordamos a formação para a pesquisa, a pesquisa como instrumento de avanços da prática, a socialização dos seus resultados, mediante o resgate da produção no Sistema Lattes do CNPq de 444 mestres egressos de cursos de mestrado acadêmico em enfermagem, diplomados entre 1995 e 2005, em universidades públicas da região sul do Brasil, constatando que 139 mestres não apresentaram qualquer publicação; e a aplicação dos resultados da pesquisa à prática. A produção de conhecimentos na enfermagem é uma estratégia para alcançar avanços na sua prática profissional e, neste processo, destaca-se a dimensão ética, desde a definição do problema de pesquisa, a necessária socialização dos achados e sua incorporação à prática profissional.
\end{abstract}

PALAVRAS-CHAVE: Pesquisa; Enfermagem; Ética.

\section{IMPACT OF THE RESULTS OF NURSING RESEARCH ON PROFESSIONAL PRACTICES}

ABSTRACT: Taking into account our concern over the results of research carried out in Nursing, this paper aims to reflect the impact that this research makes on professional practices. Therefore, we have approached the researchers' education process for research, the research itself as a tool to improve practice, and the socialization of their results by the search of the production in CNPq Lattes System of 444 nurses with master's degree qualified on public universities of South Region between 1995 and 2005, and the application of research results to practice. We identified that 139 nurses with master's degree did not presented any publication. The knowledge production in Nursing is a strategy to improve its professional practices. In this process, emphasis is given to the ethical dimension, starting from the definition of the research question, the need to socialize the findings, and their incorporation into professional practices.

KEYWORDS: Research; Nursing; Ethics.

\section{IMPACTO DE LOS RESULTADOS DE LAS INVESTIGACIONES EN ENFERMERÍAEN LA PRÁCTICA PROFESIONAL}

RESUMEN: Frente a la preocupación con los resultados de la investigación en enfermería, nuestro objetivo es reflexionar sobre su impacto en la práctica profesional. Para esto, abordamos la formación para la investigación, la investigación como instrumento de los avances de la práctica, la socialización de sus resultados, mediante el rescate de la producción en el Sistema Lattes del CNPQ de 444 maestros egresados de cursos de maestría académica en enfermería, diplomados entre 1995 y 2005, en universidades públicas de la Región Sur de Brasil, constatando que 139 maestros no presentaron alguna publicación; y la aplicación de los resultados de la investigación a la práctica. La producción de conocimientos en enfermería es una estrategia para alcanzar progresos en la práctica profesional y, en este proceso, se destaca la dimensión ética, desde la definición del problema de investigación, la necesaria socialización de los hallados y su incorporación a la práctica profesional.

PALABRAS CLAVE: Investigación; Enfermería; Ética.

\footnotetext{
${ }^{1}$ Professor Associado II da Universidade Federal do Rio Grande-FURG.

${ }^{2}$ Professor Associado II da Universidade Federal do Rio Grande-FURG.

${ }^{3}$ Professor Adjunto I da Universidade Federal do Rio Grande-FURG.

${ }^{4}$ Professora Titular da Universidade Federal de Santa Catarina-UFSC.

${ }^{5}$ Professora Titular da Escola de Enfermagem de Ribeirão Preto da Universidade de São Paulo-EERP-USP

${ }^{6}$ Aluna do Curso de Mestrado do Programa de Pós-Graduação em Enfermagem-FURG.
}

Autor correspondente:

Valéria Lerch Lunardi

Rua Dr Lavreira, 167 - 96216-040 - Rio Grande-RS

E-mail: vlunardi@terra.com.br

Recebido: 03/09/08

Aprovado: 20/02/09 


\section{INTRODUÇÃO}

Atualmente vivenciamos um contínuo e significativo crescimento da pesquisa em enfermagem como resultado tanto da formação de pesquisadores e da sua produção em grupos de pesquisa quanto da sua valorização, embora ainda carente de maior divulgação e implementação de seus resultados. Há um relevante corpo de conhecimentos já existente e permanentemente sendo produzido para trazer respostas às problemáticas que cotidianamente emergem da prática da enfermagem, das implicações deste trabalho para o processo de viver e morrer dos sujeitos.

Portanto, nos últimos anos, de modo crescente, vimos instrumentalizando-nos para a pesquisa, instigados a olhar para a nossa realidade pessoal e profissional, perguntando-nos se o que nos disseram que deveria ser ou o que é, necessita ser assim(1). Uma questão que poderíamos trazer é se os saberes que temos produzido na área da enfermagem/saúde têm contribuído para transformar a realidade que vivemos? Assim, o objetivo deste artigo é refletir sobre o impacto da pesquisa em enfermagem, abordando a formação para a pesquisa, a pesquisa como instrumento de avanços da prática, a socialização dos resultados e sua aplicação à prática.

\section{FORMAÇÃO PARA A PESQUISA}

A qualificação de enfermeiros tem sido crescente: temos, na área da enfermagem, 32 programas de pós-graduação stricto sensu, com 31 cursos de mestrado e 14 de doutorado ${ }^{(2)}$; um grande contingente de cursos de especialização, mais de 700 cursos de graduação em enfermagem; além da possibilidade de cursarmos pós-graduações stricto e lato sensu em áreas afins.

A iniciação para a pesquisa vem sendo estimulada, já na graduação, antes mesmo das proposições presentes nas atuais Diretrizes Curriculares $^{(3)}$, pela crescente atuação de estudantes em iniciação científica; pela participação em cursos de pós-graduação, em especial cursos stricto sensu, mediante a formação para a pesquisa e inserção em grupos de pesquisa, tendo em vista a atuação como futuros mestres e doutores no mercado de trabalho em instituições de ensino, assistenciais e de pesquisa. Assim, a partir de uma suposta iniciação/instrumentalização para a pesquisa em diferentes níveis, poderíamos perguntarnos se nós, enfermeiras, nos diferentes espaços em que nos inserimos, estamos produzindo conhecimentos? A produção do saber e sua aplicação na enfermagem deve ser um dever e/ou expressão de nossa autonomia no exercício da profissão?

\section{PESQUISA COMO INSTRUMENTO DE AVANÇO DA PRÁTICA}

A pesquisa "tem um vital e importante papel na nossa sociedade"; por meio dela, é que "descobertas são feitas, idéias são confirmadas ou refutadas, eventos controlados ou previstos e teorias desenvolvidas ou refinadas. Todas estas funções contribuem para o desenvolvimento do conhecimento"(4:1). Assim, se a pesquisa pode ser entendida como instrumento para a produção de conhecimento, para o conhecimento do ser humano e da sua realidade, para a sua compreensão e para a busca de soluções aos problemas e conflitos enfrentados ${ }^{(5-9)}$, esta concepção vai ao encontro do entendimento de ética como a percepção de conflitos, ou seja, a problematização do que vivemos, presenciamos e construímos, pelo exercício da autonomia e pela coerência de valores ${ }^{(10)}$.

Deste modo, nos diferentes espaços em que atuamos e nas diferentes práticas que exercemos, seja na docência, na assistência ou em atividades administrativas, na rede básica, em instituições hospitalares ou não, são diversas as situações vivenciadas que não apenas permitem, mas exigem sua problematização, tendo em vista o cuidado ao ser humano e simultaneamente o respeito à sua condição de ser humano: nas relações com os clientes, com suas famílias, com a equipe de enfermagem e de saúde, com as administrações das instituições, com a profissão, com a sociedade. É possível, no entanto, que muitas destas situações já possam fazer parte de cenários de normalidade, no nosso ambiente de trabalho, aceitos como inquestionáveis, apesar de poderem constituirse transgressões ao que um dia acreditamos como valor, como desejável e como o que entendemos por cuidado de enfermagem.

Daí, a permanente identidade possível e necessária entre o nosso cotidiano de trabalho na saúde e na enfermagem e a ética, ou seja, a dimensão ética do trabalho da enfermagem e a dimensão ética do cuidado de enfermagem: a permanente interface entre a ética no trabalho da enfermagem e o questionamento acerca deste. Continuamente, frente ao modo como cuidamos e vemos outros serem cuidados, podemos nos perguntar se é este o modo como deveríamos cuidar ou como as pessoas deveriam ser cuidadas? 
Tais questionamentos possibilitam nos remeter permanentemente à uma reflexão sobre os fundamentos éticos que são a base para a construção do saber da enfermagem: a dimensão ética do cuidado de enfermagem, a dignidade humana, o respeito à vida, à justiça e à qualidade do cuidado. Neste sentido, é possível entender a pesquisa não apenas como mais uma das dimensões do trabalho da enfermagem, mas como um instrumento/uma estratégia que contribui fortemente para uma atuação ética, pelo seu permanente estímulo a um olhar de questionamento da realidade e de possível inconformidade com o instituído, em que "o conhecimento deve [...] mobilizar a atitude geral do espírito humano para propor e resolver problemas”(11:19).

Frente aos problemas detectados no ambiente de trabalho e que podem comprometer as ações de cuidado ao ser humano, a enfermeira tanto pode permanecer indiferente como, a partir de um questionamento interior, pode não apenas posicionarse diante do que percebe, mas, demonstrando seu inconformismo e insatisfação, mobilizar-se, em busca do conhecimento já produzido, de tecnologias de cuidado e/ou para produzir conhecimentos, na tentativa de encontrar respostas que permitam mudanças na realidade e qualificação do cuidado de enfermagem ${ }^{(12)}$.

A questão é se, tendo em vista a formação e a preparação para atuar em pesquisa, estamos formando enfermeiras, mestres e doutoras como cidadãs capazes de enfrentar os problemas de nosso tempo? E mais ainda, que realidade se pode construir e mobilizar para que os avanços sejam de um melhor sempre e de um além que ultrapasse a solução de problemas e busque um novo, além do esperado ${ }^{(11)}$ ?

\section{SOCIALIZAÇÃO DOS RESULTADOS DA PESQUISA}

Na realização do mestrado e doutorado, a elaboração de uma dissertação e de uma tese, respectivamente, predominantemente sob a forma de uma pesquisa, constitui-se uma exigência para a titulação, assim como vem sendo estimulada e exigida a produção científica da pós-graduanda e da egressa, pela sua relevância e expressão do compromisso com a sociedade, como uma das retribuições pelo investimento social em sua formação, o seu próprio compromisso com a instituição formadora e com o seu processo de avaliação. No entanto, as pósgraduandas, já durante sua formação em pósgraduação stricto sensu e após sua titulação, estão demonstrando compromisso com a enfermagem e a sociedade, no que se refere à produção científica? Terão a compreensão de que a difusão e a aplicação de conhecimentos é uma responsabilidade ética?

Assim, com vistas a conhecer aspectos da socialização/divulgação dos resultados da pesquisa em enfermagem, optamos por resgatar a produção de mestres egressos de cursos de mestrado acadêmico em enfermagem, diplomados entre 1995 e 2005, em universidades públicas da região sul quanto a sua produção anterior ao ano de sua titulação como mestres e após a mesma, bem como, a sua atuação como docentes ou não, em instituições públicas e não públicas; e a realização ou não de doutorado.

As universidades da região sul, cuja produção científica de suas egressas foi buscada, são: a Universidade Federal de Santa Catarina-UFSC, programa que se iniciou em 1976 e, portanto, a análise foi feita de 1995 a 2005; a Universidade Federal do Rio Grande do Sul-UFRGS, iniciado em 1998, cuja análise foi de 2000 a 2005; a Fundação Universidade Federal do Rio Grande-FURG, que se iniciou em 2002, analisando-se de 2003 a 2005 e a Universidade Federal do Paraná-UFPR, cujas atividades no Programa de Pós- Graduação em Enfermagem iniciaram em 2003 e a análise foi de 2004 a 2005.

Assim, a partir de informações provenientes destes Programas, houve a formação de um total de 444 egressos de cursos de mestrado em enfermagem, sendo 419 (94,3\%) mulheres e 25 (5,7\%) homens, dos quais 302 egressos da UFSC (68,0\%), 97 da UFRGS (21,8\%), 25 da FURG (5,6\%) e 20 da UFPR (4,5\%), assim distribuídos: 1995 - 9 egressos; 1996 - 19; 1997 - 23; 1998 - 39; 1999 - 33; 2000 - 95; 2001 - 28; 2002 - 54; 2003 - 36; 2004 - 61; 2005 - 46 egressos.

Com o propósito de realizar uma análise documental, foram buscados os curricula vitae destes 444 egressos, no Sistema Lattes do CNPq, tendo sido encontrados os curricula de 407 (91,7\%) egressos. Os dados foram analisados através do software Statistical SPSS.

Destes 407 egressos, 301 (74\%) atuam como docentes, assim distribuídos: 128 (42,5\%) egressos, em instituições de ensino superior federais; 8 (2,7\%) em instituições de ensino estaduais; 162 ( 53,8\%) em instituições de ensino não públicas e 3 (1\%) atuam em escolas de primeiro e segundo graus, não na área da enfermagem. Os demais 106 (26\%) egressos, que não desenvolvem atividades como docentes, atuam em instituições de saúde, públicas e privadas, hospitais, 
secretarias de saúde. Dentre os 407 egressos, 26 (6,3 $\%)$ atuam tanto na docência como em atividades assistenciais.

Desse total de 407 mestres formados, 47 são doutores e 61 estão cursando o doutorado. Dentre estes doutores, 35 (76,1\%) atuam em instituições de ensino públicas e 11 (23,9\%) nas outras instituições de ensino. Dos demais mestres, 134 (65\%) estão nas instituições de ensino não públicas e 69 (33\%) nas públicas. Dos mestres que estão realizando o doutorado, 32 (65,3\%) estão em instituições de ensino públicas e 17 (34,7\%) nas outras instituições de ensino não públicas.

Em busca da produção de artigos em periódicos, de capítulos e livros, consideramos apenas as produções científicas descritas de forma completa, constando, inclusive, as páginas da publicação, independentemente de sua classificação no sistema Qualis. Relacionando a produção dos egressos antes de sua titulação como mestres e seu ambiente de atuação, não constatamos diferença significativa: os que não atuavam na docência tiveram uma média de 1,31 publicações/ano e os que atuam em docência em instituições de ensino e em outros ambientes de trabalho, uma média de 1,17 publicações/ano; os que atuam nas instituições de ensino públicas, tiveram uma média de 1,23 publicações/ano e, nas não públicas, 1,24 publicações/ano, cujo $\mathrm{p}<0,78$.

Após a conclusão do mestrado, constatamos que, dentre os 361 egressos até 2004, os que atuavam na docência, em instituições de ensino públicas, apresentaram uma média de 0,87 publicações/ano, enquanto que os de instituições de ensino não públicas tiveram uma média de 0,49 publicações/ano. Utilizandose o teste T de Student, estas diferenças mostraram-se estatisticamente significativas (99\% de certeza de que nas públicas, docentes publicam mais). Já os que não atuam em atividades de docência, apresentaram 0,62 produções/ano como média e os que atuam em atividades de docência e em outros ambientes de trabalho, tiveram uma média de 0,52 produções/ano.

Após a sua titulação, as mestras que atuam como docentes em instituições públicas tiveram uma média de 5,57 publicações/ano e aquelas que atuam nas instituições não públicas tiveram 2,21 publicações/ ano. Ainda, dos 222 egressos que tiveram publicação, 46 mestres, que correspondem a 20,72\% da amostra, produziram $57,8 \%$ de toda esta produção, o que demonstra uma concentração das publicações em um pequeno grupo de mestres.

A partir do ano da titulação, identificamos que, dos 361 egressos (46 se titularam em 2005), 139 mestres (38,5\%) não apresentaram qualquer publicação. Destes 139 egressos, 3 (6,4\%) são mestres, que já concluíram o doutorado, porém também não apresentaram publicação; a partir da conclusão do mestrado, 5 (8,8\%), que cursavam o doutorado não tiveram publicação e os demais 131 (51,0\%) mestres não publicaram.

Com base nesta exposição, várias questões podem ser trazidas. Constatamos que, nos últimos 10 anos, na área da enfermagem na Região Sul, 139 mestrandos concluíram sua pós-graduação e não tiveram qualquer produção na área, sendo que 30 (23\%) deles estão em universidades públicas, 61 $(41,5 \%)$ em outras instituições de ensino e 48 atuam em outros ambientes. Apesar dos benefícios inquestionáveis dos conhecimentos já produzidos na área da enfermagem, surpreende-nos o fato de que alguns mestres e, também doutores, após sua titulação, não vêm divulgando o conhecimento produzido.

A busca do conhecimento sem a divulgação do conhecimento produzido é ética? Entendemos que a difusão e aplicação dos conhecimentos é uma responsabilidade ética, fortemente associada ao exercício de autonomia das próprias profissionais. Após a titulação stricto sensu, cabe também a responsabilidade de prosseguir a produção de conhecimentos e orientar ações que possam responder as exigências da realidade da enfermagem. Ainda, a socialização dos conhecimentos é fundamental para a construção dos valores e comportamentos desejados.

Por outro lado, como docentes, sabemos das inúmeras dificuldades que temos, atualmente, para contemplar atividades de ensino, freqüentemente não apenas na graduação, além da pesquisa e extensão; os múltiplos compromissos a que somos estimulados e pressionados a assumir. Dentre a produção científica possível de ser publicada, sequer os produtos dos trabalhos de conclusão de curso que orientamos podem não estar sendo suficientemente socializados, ficando limitados ao acervo de nossas próprias instituições, nas estantes das bibliotecas.

A questão, então, parece ser a necessidade de não apenas problematizarmos o cotidiano do trabalho e estimularmos o olhar crítico das estudantes como práticas autônomas, mas implementar investigações e socializarmos resultados e avanços que contribuam para a transformação de nossas realidades. Nós, como docentes de universidades públicas ou não, enfermeiras que atuamos em diferentes espaços, instituições de saúde, de educação, sabemos que a exigência de 
produção científica habitualmente não se situa no âmbito institucional. Em grande parte das instituições de ensino, até pode haver um estímulo para a qualificação dos docentes ao mestrado e doutorado, porém não necessariamente há um estímulo ou exigência de produção científica, diferentemente de quando as docentes participam de programas de pósgraduação stricto sensu.

A produção científica da docente se encontra fortemente situada no âmbito da sua autonomia, de priorizar o seu tempo para esta atividade. Este processo, todavia, pode constituir-se primeiramente em um embate interno, da própria profissional, de motivação, de compreensão e aceitação da necessidade de produção, de vontade e desejo de produzir, de reconhecer a necessidade de priorização do seu tempo de trabalho para esta atividade; e, frequentemente, de embates externos, com chefias, com seus pares, de resistir a outras possíveis prioridades institucionais, exigindo, por vezes, quase que permanentemente, condições de trabalho para esta produção tais como a priorização de um número de horas de trabalho para atividades de produção científica, a concessão de bolsas, justificando o que parece o óbvio: por que se qualificar? Por que cursar um mestrado? Por que cursar um doutorado? Qual a sua finalidade? Para comprovar apenas a titulação docente exigida nos cursos de graduação em enfermagem, frente a processos de avaliação externos?

Para demonstrar uma qualificação diferenciada nos cursos de graduação e nas instituições de saúde, parece necessário, para além do título, a produção de conhecimento e a aplicação do conhecimento produzido, a partir do questionamento do vivido na área da saúde e da enfermagem e sua conseqüente socialização; ações que, mesmo que desencadeadas em grupos de pesquisa e grupos de trabalho, estão bastante relacionadas com o movimento inicial de vontade, de autonomia dos sujeitos, pois ainda não há, comumente, como já dito, exigência externa de produção científica aos docentes e enfermeiras. Assim, os conhecimentos produzidos estão alcançando o público desejado?

Aplicação dos resultados da pesquisa à prática Para finalizar, e articulando com a ampliação vertiginosa de cursos de graduação em todo o Brasil, o número crescente de formação de mestres e sua absorção em muitos destes cursos, parece necessário questionar acerca da qualidade do cuidado de enfermagem que, cotidianamente, vem acontecendo nas instituições de saúde. Uma questão fortemente enfatizada no último Seminário Nacional de Pesquisa em Enfermagem-SENPE, em Florianópolis, 2007, referiu-se à problemática da incorporação dos resultados de pesquisa às práticas de saúde implementadas, da necessidade de construirmos estratégias para transferirmos e aplicarmos tais resultados aos campos de prática. Estaremos sendo consumidores de pesquisas, aplicando em nosso fazer o que vimos produzindo como saber da enfermagem ao fazer da enfermagem, de modo a realmente contribuir para a qualificação deste cuidado e deste fazer, valorizando a produção da área?

A pesquisa liga teoria, educação e prática. Formulações teóricas apoiadas em achados de pesquisas podem potencialmente se tornar o fundamento da prática de enfermagem $^{(13: 5)}$.

Enfermeiras com formação de mestrado e doutorado, além de pesquisadoras e coordenadoras de pesquisa necessitam ser "consumidores sofisticados de pesquisas". Enfermeiras assistenciais ocupam espaços privilegiados geradores de problemas e questões de pesquisas, necessitando mobilizar-se para a busca de respostas a estes questionamentos, participando de estudos científicos ${ }^{(13: 6)}$.

No entanto, dentre as principais barreiras apontadas pelas enfermeiras para a utilização de resultados de pesquisas em sua prática assistencial, destacam-se “a precariedade de tempo para implementar novas idéias, falta de conhecimento sobre resultados de pesquisa em enfermagem, falta de acesso à literatura relevante"(14:1). Neste sentido, parece pertinente o estudo ${ }^{(15)}$ que, frente à constatação da inexpressiva atividade de pesquisa por parte das enfermeiras, implementaram práticas educativas em metodologia da pesquisa com 86 enfermeiras assistenciais do Hospital Universitário da UFPR (de um total de 202, correspondendo a 42,5\%) com a finalidade de estimulá-las "a participarem do universo da pesquisa, auxiliá-las na organização metodológica de idéias, conhecimentos e experiências"(15:358), tendo sido concluídos 70 projetos, dos quais 28 foram implementados, com divulgação de resultados em anais de eventos ou em periódicos indexados.

Dentre as justificativas apontadas para a inconclusão dos projetos e/ou seu não desenvolvimento, foram apontadas: dificuldades de articular teoria e prática e de redigir o projeto; questões organizacionais, requerendo uma maior disponibilidade de tempo da enfermeira para o seu enfrentamento (falta de pessoal, material e equipamentos), com a não priorização do 
tempo para as atividades relativas ao projeto, além da falta de leitura do material disponibilizado, de bibliografia, dificuldades relacionadas a traduções, uso do computador, falta de tempo fora do ambiente de trabalho.

Em outro estudo(16), entrevistas com 35 pesquisadores seniors de serviços de saúde, financiadores de pesquisa e gerentes dos serviços de saúde demonstraram sua insatisfação com o impacto das pesquisas nos serviços de saúde, sob diferentes perspectivas: pesquisadores estão frustrados com o pequeno uso do produto de seu trabalho por gestores e gerentes, sentindo que o conhecimento por eles gerado é pouco valorizado e pouco aplicado; administradores percebem pouca relevância nas pesquisas realizadas, considerando, o valor financeiro investido, um desperdício; gestores preocupam-se com a questão temporal, entendendo que os resultados das pesquisas deveriam estar disponíveis, quando decisões políticas fossem tomadas; todos os grupos reconhecem a necessidade urgente de articulação entre os financiadores de pesquisas, os que as utilizam e os que as produzem.

Os autores perguntam:

quanto das informações geradas nas pesquisas têm sido transferidas e utilizadas para subsidiar decisões práticas sobre saúde e cuidado em saúde? A pesquisa está perseguindo uma correta agenda? Está examinando as questões adequadas, do modo correto? Estão os resultados sendo comunicados às pessoas que os necessitam $^{(16: 1340)}$ ?

No entanto, gestores, pesquisadores e usuários de pesquisa parecem unidos na crença de que não lhes cabe a responsabilidade por disseminar os resultados de pesquisa e o apoio para a sua aplicação.

De outro modo, a cultura de nossos profissionais no exercício de uma prática não articulada à produção ou, até mesmo, ao consumo de novos conhecimentos parece ser uma assertiva bastante questionada. Assim, como o cidadão pode estar recebendo um cuidado mais atualizado? De quem é a responsabilidade da aplicação ou utilização de novos conhecimentos na prática da profissão?

A distinção entre a educação acadêmica e escolar de tantas outras maneiras de educar faz-se porque a educação acadêmica e escolar baseiam-se "no processo de pesquisa e formulação própria”(17:1). A pesquisa tem como diferencial o questionamento reconstrutivo, que engloba teoria e prática, qualidade formal e política, inovação e ética. Deste modo, as enfermeiras como profissionais da educação, nos diferentes âmbitos em que atuam, precisam ser pesquisadoras, reconhecendo e trabalhando "a pesquisa como princípio educativo e como atividade cotidiana, atuando não como profissionais da pesquisa, mas como profissionais da educação pela pesquisa” ${ }^{17: 2)}$.

\section{PARA FINALIZAR}

Sabemos que o tema é bastante complexo e não temos subsídios suficientes para sustentar algumas respostas para as questões aqui apresentadas. À guisa de finalização, ousamos sim apontar outros questionamentos: mas afinal, qual é o impacto real dos conhecimentos que estamos produzindo, socializando e utilizando na prática do cuidado em enfermagem e saúde? Estes conhecimentos são utilizados por outros profissionais e cidadãos? A formação dos pesquisadores para a pesquisa inclui o compromisso com o impacto na aptidão para pesquisar e socializar os conhecimentos que produz?

Reconhece-se que o processo de produção de conhecimentos na enfermagem é uma importante estratégia para avanços da prática de enfermagem e saúde e que a dimensão ética neste processo remete à autonomia do pesquisador na definição do problema de pesquisa, na necessária socialização dos achados e na sua incorporação à prática profissional, extrapolando a objetividade em busca sempre de um melhor cuidado e um melhor viver humano. Eis o nosso grande desafio!

\section{REFERÊNCIAS}

1. Foucault M. A história da sexualidade II. O uso dos prazeres. Rio de Janeiro: Graal; 1984.

2. Ministério da Educação (BR). CAPES. Cursos recomendados [Internet]. Brasília (DF):CAPES; 2008 [citado 2008 Jun 25]. Disponível: www.conteudoweb.capes.gov.br.

3. Ministério da Educação (BR). Secretaria de Educação Superior. Diretrizes curriculares para os cursos de graduação. Resolução CNE/ CES Nº 3, de 7 de novembro de 2001 [online]. Disponível: www.mec.gov.br/sesu/ diretriz.htm [Acesso em 28 maio 2005].

4. Morse J. Critical issues in qualitative research methods. Thousand Oaks, London, New Delhi: Sage Publications; 1994.

5. Lüdke M, André M. Pesquisa em educação: abordagens 
qualitativas. São Paulo: EPU; 1986.

6. Pádua EMM. Metodologia da pesquisa: abordagem teórico-prática. 10ª ed. Campinas: Papirus; 2004.

7. Chizzotti A. Pesquisa em ciências humanas e sociais. $9^{a}$ ed. São Paulo: Cortez; 2005.

8. Trentini M, Paim L. Pesquisa em enfermagem - uma modalidade convergente-assistencial. $2^{\text {a }}$ ed. Florianópolis: UFSC; 2004.

9. Rea LM, Parker RA. Metodologia da pesquisa: do planejamento à execução. São Paulo: Pioneira; 2002.

10. Segre M, Cohen C. Bioética. São Paulo: EdUSP; 1995.

11. Morin E, Almeida MC, Carvalho EA. Educação e complexidade. Os setes saberes e outros ensaios. São Paulo: Cortez; 2002.

12. Trentini M, Beltrame V. A pesquisa convergenteassistencial (PCA) levada ao real campo de ação da enfermagem. Cogitare Enferm. 2006 Mai/Ago;11(2):15660 .

13. Liobondo-Wood G, Haber J. Pesquisa em enfermagem: métodos, avaliação crítica e utilização. Rio de Janeiro: Guanabara Koogan; 2001.

14. Carroll DL, Greenwood R, Lynch KE, Sullivan JK, Ready C, Fitzmaurice JB. Barriers and facilitators to the utilization of nursing research. Clinical Nurse Specialist. 1997;11(5):207-12.

15. Dyniewicz AM, Gutiérrez MG. Metodologia da pesquisa para enfermeiras de um hospital universitário. Rev Latino-Am Enferm. 2005;13(3):354-63.

16. Dash P, Gowman N, Traynor M. Increasing the impact of health services research. British Medical Journal. 2003;327:1339-41.

17. Demo P. Educar pela pesquisa. $2^{\mathrm{a}}$. Ed. Campinas: Autores Associados; 1997. 